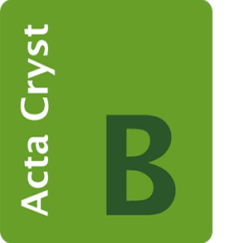

STRUCTURAL SCIENCE

CRYSTAL ENGINEERING

MATERIALS

Volume 74 (2018)

Supporting information for article:

Crystal Packing Control of Trifluoromethyl-Substituted Furan/Phenylene Co-oligomer

Alina A. Sonina, Igor P. Koskin, Peter S. Sherin, Tatyana V. Rybalova, Inna K. Shundrina, Evgeny A. Mostovich and Maxim S. Kazantsev 


\section{S1. Characterization}

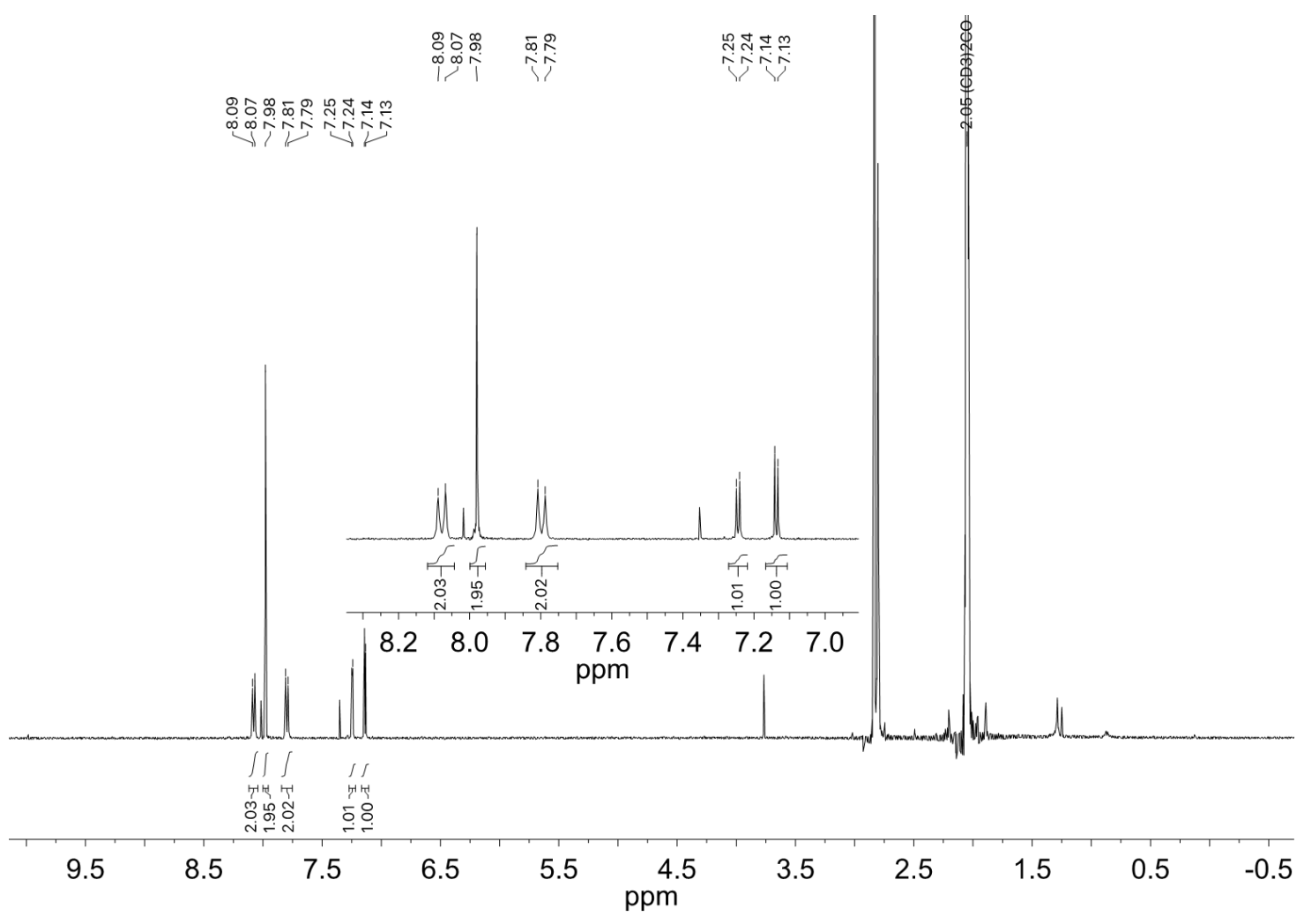

Figure S1 ${ }^{1} \mathrm{H}$ NMR spectrum of 1,4-bis(5-(4-(trifluoromethyl)phenyl)furan-2-yl)benzene in $\left(\mathrm{CD}_{3}\right)_{2} \mathrm{CO}$.

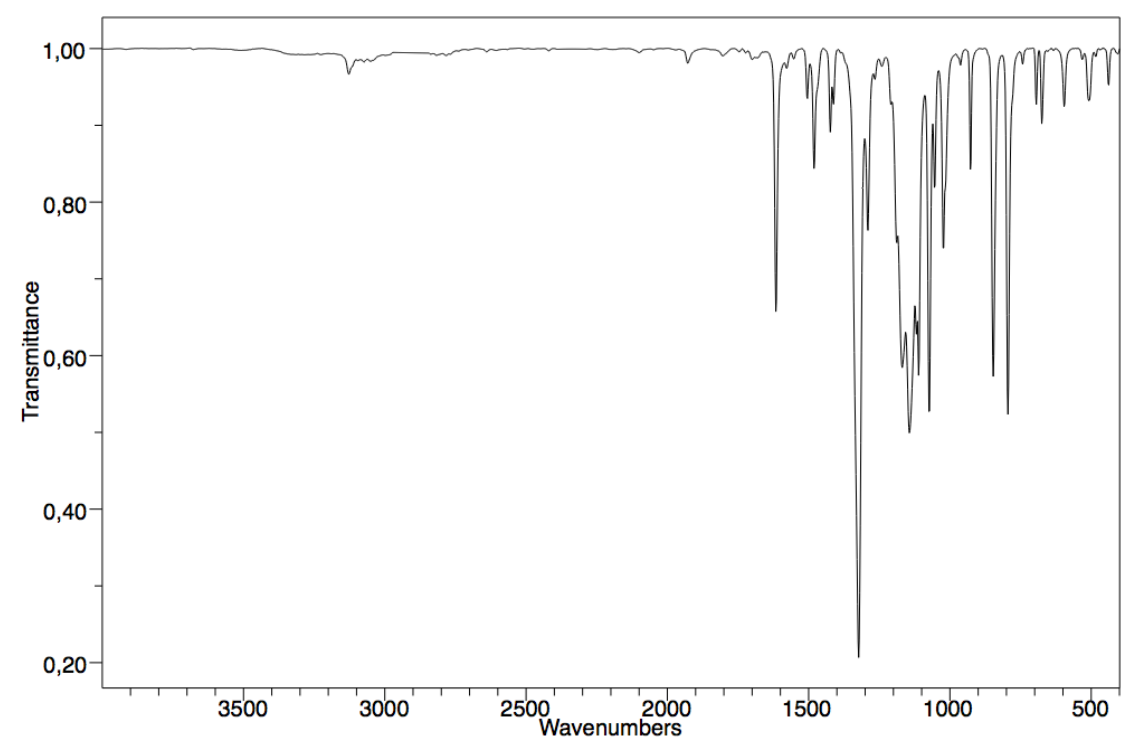

Figure S2 FT IR spectrum of 1,4-bis(5-(4-(trifluoromethyl)phenyl)furan-2-yl)benzene in KBr. 


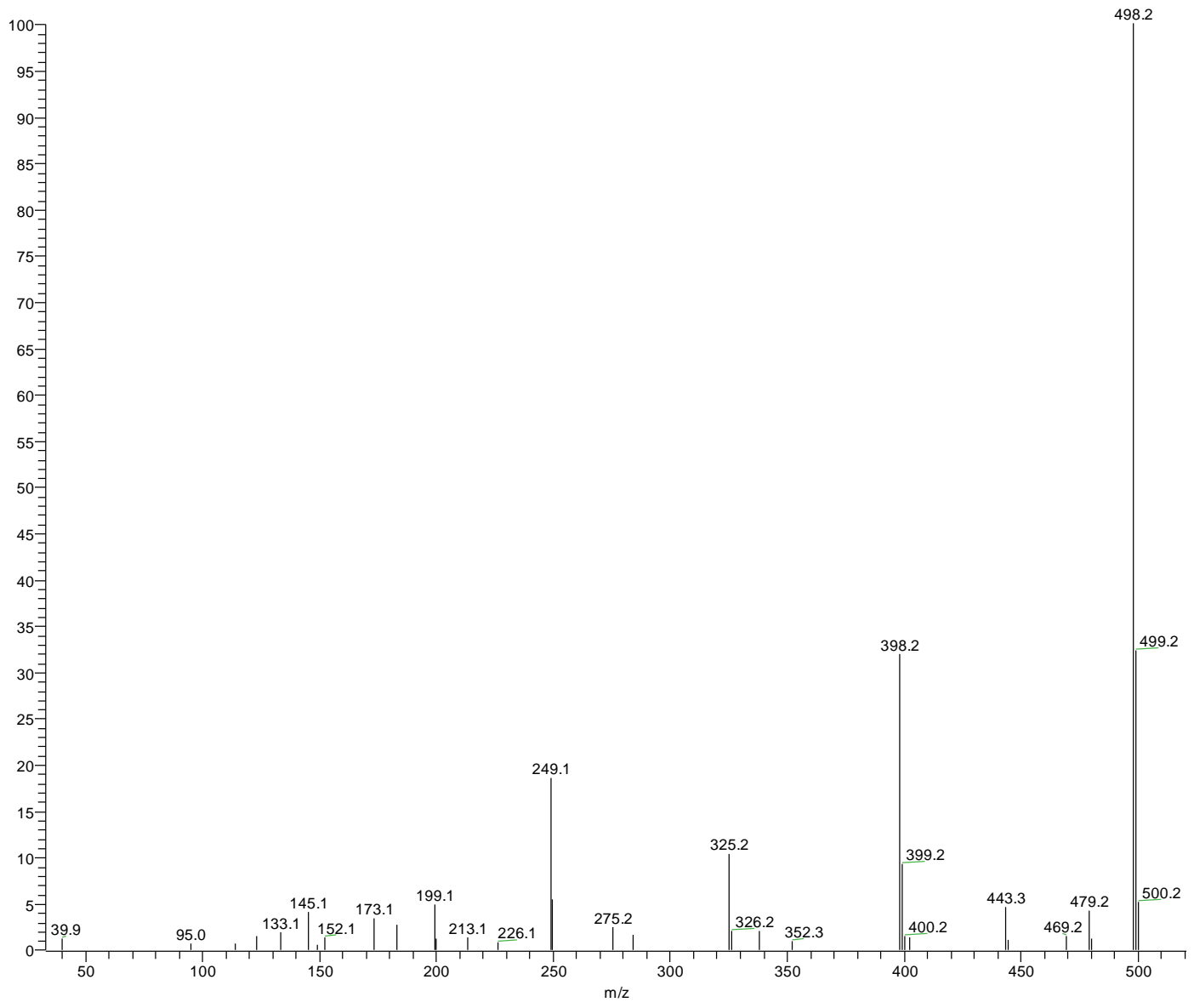

Figure S3 HRMS spectrum of 1,4-bis(5-(4-(trifluoromethyl)phenyl)furan-2-yl)benzene. 
a)

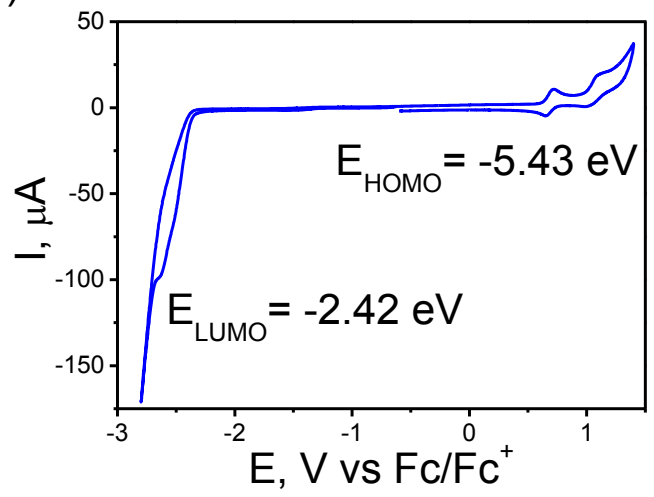

C)

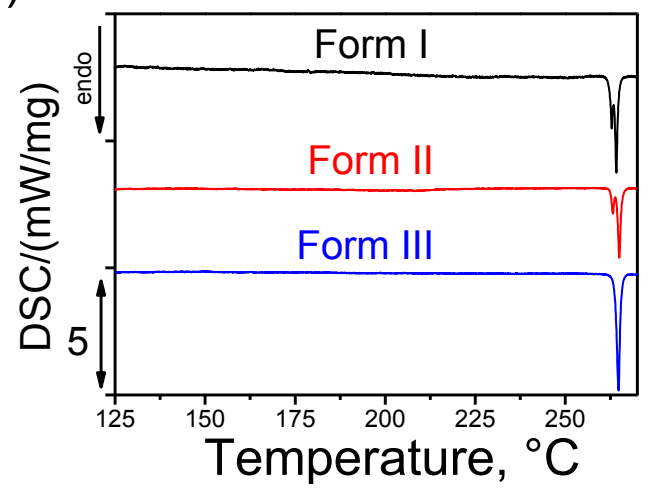

b)

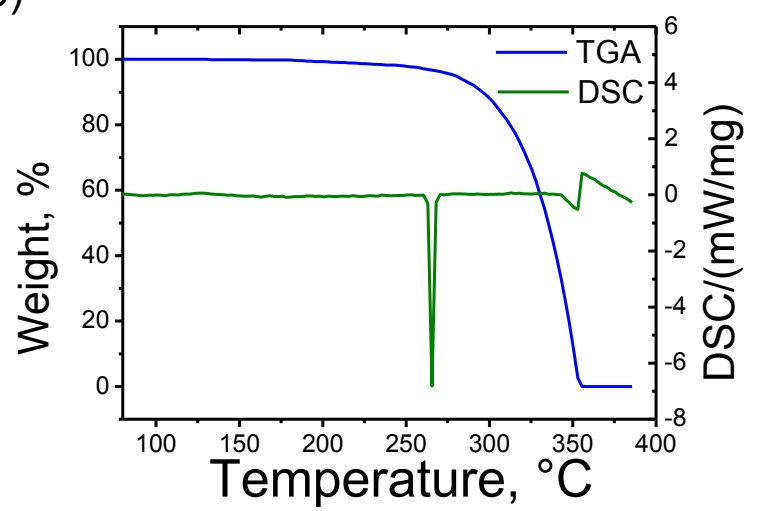

Figure S4 a) Cyclic voltammogramm of $\mathrm{CF}_{3}-\mathrm{BPFB}$ in $\mathrm{CH}_{2} \mathrm{Cl}_{2}$ solution; b) Thermal gravimetric and differential scanning calorimetric analyses of $\mathrm{CF}_{3}$-BPFB powder in $\mathrm{He}$ atmosphere; c) Differential scanning calorimetric analyses of $\mathrm{CF}_{3}$-BPFB polymorphs in $\mathrm{He}$ atmosphere.

\section{S2. X-ray data}

Table S1 Crystal data, data collection and structure refinement parameters for crystal structures of $\mathrm{CF}_{3}$-BPFB.

I $\quad$ II $\quad$ III

\begin{tabular}{|c|c|c|c|}
\hline Chemical formula & \multicolumn{3}{|c|}{$\mathrm{C}_{28} \mathrm{H}_{16} \mathrm{~F}_{6} \mathrm{O}_{2}$} \\
\hline$M_{\mathrm{r}} / \mathrm{g} \cdot \mathrm{mol}^{-1}$ & \multicolumn{3}{|c|}{498.41} \\
\hline $\begin{array}{c}\text { Crystal system, } \\
\text { space group }\end{array}$ & \multicolumn{2}{|r|}{ Monoclinic, $P 2_{1} / c$} & \\
\hline Z & 2 & 2 & 8 \\
\hline Crystal size (mm) & $0.25 \times 0.24 \times 0.008$ & $0.60 \times 0.18 \times 0.05$ & $1.00 \times 0.12 \times 0.02$ \\
\hline & $23.238(4)$ & $11.505(5)$ & $20.544(1)$ \\
\hline
\end{tabular}




\begin{tabular}{|c|c|c|c|}
\hline \multirow[t]{2}{*}{$a, b, c(\AA)$} & $7.529(1)$ & $6.2350(6)$ & $6.2569(3)$ \\
\hline & $6.2437(9)$ & $16.012(2)$ & $35.797(2)$ \\
\hline$\beta\left(^{\circ}\right)$ & $92.830(6)$ & $109.293(6)$ & $106.649(3)$ \\
\hline$V\left(\AA^{3}\right)$ & $1091.1(3)$ & $1084.1(2)$ & 4408.4(4) \\
\hline$D_{\text {calc }} / \mathrm{g} \cdot \mathrm{cm}^{-3}$ & 1.517 & 1.527 & 1.502 \\
\hline$\mu\left(\mathrm{mm}^{-1}\right)$ & 0.13 & 0.13 & 0.13 \\
\hline No. of measured, & 8904, & 8382 , & 50554 \\
\hline $\begin{array}{c}\text { independent and } \\
\text { observed }[I>2 \sigma(I)]\end{array}$ & 1882 & 1919, & 7767 \\
\hline reflections & 1221 & 1436 & 3615 \\
\hline$R_{\text {int }}$ & 0.048 & 0.023 & 0.093 \\
\hline$\theta_{\min }\left({ }^{\circ}\right)$ & 3.225 & 3.535 & 1.035 \\
\hline$\theta_{\max }\left({ }^{\circ}\right)$ & 24.876 & 25.027 & 25.027 \\
\hline \multicolumn{4}{|l|}{ Range of } \\
\hline$h$ & $-27 \rightarrow 25$ & $-13 \rightarrow 13$ & $-24 \rightarrow 24$ \\
\hline$k$ & $-8 \rightarrow 8$ & $-7 \rightarrow 7$ & $-7 \rightarrow 7$ \\
\hline$l$ & $-7 \rightarrow 7$ & $-18 \rightarrow 19$ & $-42 \rightarrow 42$ \\
\hline$R 1, w R\left(F^{2}\right), S$ & $0.042,0.093,1.05$ & $0.032,0.087,1.03$ & $0.060,0.193,1.06$ \\
\hline No. of parameters & 190 & 190 & 757 \\
\hline$\Delta \rho_{\max }, \Delta \rho_{\min }\left(\mathrm{e} \AA^{-3}\right)$ & $0.13,-0.17$ & $0.14,-0.17$ & $0.23,-0.21$ \\
\hline
\end{tabular}

Table S2 The distances and angles of noncovalent interactions $\mathrm{C}_{\mathrm{sp} 2}-\mathrm{H} \cdots \pi, \mathrm{C}_{\mathrm{sp} 3}-\mathrm{F} \cdots \pi$ and $\mathrm{C}_{\mathrm{sp} 2}-$ $\mathrm{H} \cdots \mathrm{F}$ for polymorphic forms.

\begin{tabular}{|c|c|c|c|c|}
\hline form & interaction & $\mathrm{d}(\mathrm{X} \cdots \mathrm{Cg}) / \mathrm{d}(\mathrm{H} \cdots \mathrm{F}), \AA$ & $\mathrm{D}_{\mathrm{pln}} / \mathrm{d}(\mathrm{C} \cdots \mathrm{F}), \AA$ & C-X...Cg/C-H...F, \\
\hline \multirow[t]{2}{*}{$\mathrm{I}$} & $\mathrm{C} 2-\mathrm{H} \cdots \mathrm{Cg}(2)$ & 2.87 & 2.85 & 136 \\
\hline & $\mathrm{C} 8-\mathrm{H} \cdots \mathrm{Cg}(3)$ & 2.95 & 2.92 & 137 \\
\hline \multirow[t]{4}{*}{ II } & $\mathrm{C} 8-\mathrm{H} \cdots \mathrm{Cg}(2)$ & 3.00 & 2.93 & 127 \\
\hline & $\mathrm{C} 11-\mathrm{H} \cdots \mathrm{Cg}(3)$ & 3.05 & 3.01 & 143 \\
\hline & $\mathrm{F} 2(\mathrm{~F} 5) \cdots \mathrm{C} 5$ & $3.36(3.43)$ & $3.05(3.15)$ & 145 (135) \\
\hline & $\mathrm{C} 3-\mathrm{H} \cdots \mathrm{F} 1(\mathrm{~F} 4)$ & $2.78(2.75)$ & $3.65(3.62)$ & $156(157)$ \\
\hline
\end{tabular}




\begin{tabular}{|c|c|c|c|c|}
\hline & $\mathrm{C} 9-\mathrm{H} \cdots \mathrm{F} 4$ & 2.69 & 3.51 & 147 \\
\hline III & $\mathrm{C} 11^{\prime}-\mathrm{H} \cdots \mathrm{Cg}(3 \mathrm{~B})$ & 3.04 & 3.01 & 139 \\
\hline & $\mathrm{C} 2^{\prime}-\mathrm{H} \cdots \mathrm{Cg}(2 \mathrm{~A})$ & 2.89 & 2.87 & 136 \\
\hline & C8'-H $\cdots \mathrm{Cg}(2 \mathrm{~B})$ & 3.04 & 2.99 & 130 \\
\hline & $\mathrm{C} 8-\mathrm{H} \cdots \mathrm{Cg}(3 \mathrm{~A})$ & 2.90 & 2.89 & 134 \\
\hline & C8B-H $\cdots \mathrm{Cg}\left(2^{\prime}\right)$ & 3.01 & 2.97 & 128 \\
\hline & C2A-H $\cdots \mathrm{Cg}\left(2^{\prime}\right)$ & 2.92 & 2.88 & 137 \\
\hline & C8A-H $\cdots \mathrm{Cg}(3)$ & 2.87 & 2.86 & 136 \\
\hline & C2B-H‥F6 (F3) & $2.55(2.60)$ & $3.31(3.40)$ & $139(145)$ \\
\hline & C9'-H $\cdots$ F4B & 2.65 & 3.41 & 140 \\
\hline & C3-H $\cdots$ F4B & 2.71 & 3.53 & 148 \\
\hline & C2-H $\cdots$ F6B & 2.62 & 3.41 & 142 \\
\hline & C10-H $\cdots$ F6A & 2.66 & 3.22 & 119 \\
\hline & $\mathrm{F} 2 \mathrm{~B} \cdots \mathrm{C} 4$ & 3.64 & 3.15 & 141 \\
\hline & C3B-H $\cdots$ F1' & 2.76 & 3.64 & 158 \\
\hline & $\mathrm{F} 4 \cdots \mathrm{C} 3 \mathrm{~B}$ & 3.85 & 3.22 & 118 \\
\hline & $\mathrm{C} 10^{\prime}-\mathrm{H}^{\prime} \cdot \mathrm{F} 3^{\prime}$ & 2.74 & 3.26 & 117 \\
\hline & C10-H $\cdots$ F6A & 2.66 & 3.22 & 119 \\
\hline & C10A-H $\cdots$ F3A & 2.73 & 3.27 & 118 \\
\hline & C10A-H $\cdots$ F5 & 2.69 & 3.41 & 135 \\
\hline
\end{tabular}

${ }^{*} \boldsymbol{C} \boldsymbol{g}$ is the aromatic ring center; $\boldsymbol{D}_{\boldsymbol{p l n}}$ is the nearest distance between $\mathrm{X}$-atom (H/F-atom) and aromatic ring plane; $\mathrm{Cg}(1 ; 1$ '; $1 \mathrm{~A} ; 1 \mathrm{~B})$, is the furan ring; $\mathrm{Cg}\left(2 ; 2^{\prime} ; 2 \mathrm{~A} ; 2 \mathrm{~B}\right)$ is the terminal phenyl ring; $\mathrm{Cg}(3$; $3 \mathrm{~A} ; 3 \mathrm{~B}$ ) is the central phenyl ring (Fig. 1).

Table S3 The distances and the angles for F...F interactions and aggregates for polymorphic forms. ( $\theta_{1}$ and $\theta_{2}$ are the angles $\mathrm{C}_{\mathrm{sp} 3}-\mathrm{F}_{1} \cdots \mathrm{F}_{2}$ and $\mathrm{F}_{1} \cdots \mathrm{F}_{2}-\mathrm{C}_{\mathrm{sp} 3}$ of $\mathrm{C}_{\mathrm{sp} 3}-\mathrm{F}_{1} \cdots \mathrm{F}_{2}-\mathrm{C}_{\mathrm{sp} 3}$ contact).

\begin{tabular}{|c|c|c|c|c|}
\hline form & interaction & $\mathrm{d}(\mathrm{F} \cdots \mathrm{F}), \AA$ & $\theta_{1}, \stackrel{\circ}{ }$ & $\theta_{2}, \circ$ \\
\hline \multirow[t]{2}{*}{ I } & 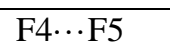 & 2.70 & 154 & 166 \\
\hline & $F 1 \cdots F 1$ & 2.70 & 158 & 158 \\
\hline
\end{tabular}




\begin{tabular}{|c|c|c|c|c|}
\hline & F2 $\cdots F 5$ & 2.87 & 127 & 104 \\
\hline & F1 $\cdots F$ F3(F6) & $3.16(3.17)$ & $99(106)$ & $121(120)$ \\
\hline & $\mathrm{F} 4 \cdots \mathrm{F} 6$ & 3.21 & 104 & 108 \\
\hline & $\mathrm{F} 3 \cdots \mathrm{F} 5$ & 3.23 & 124 & 97 \\
\hline II & 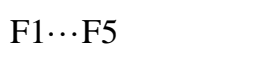 & 2.82 & 127 & 118 \\
\hline & $\mathrm{F} 6 \cdots \mathrm{F} 1(\mathrm{~F} 4)$ & $3.07(3.11)$ & $129(124)$ & $96(95)$ \\
\hline & $\mathrm{F} 1(\mathrm{~F} 4) \cdots \mathrm{F} 2(\mathrm{~F} 5)$ & $3.02(3.05)$ & 107 (122) & $126(113)$ \\
\hline III (M-M') & $\mathrm{F} 1{ }^{\cdots} \mathrm{F}^{\prime}{ }^{\prime}\left(\mathrm{F} 6^{\prime}\right)$ & $2.78(2.88)$ & $177(165)$ & $112(108)$ \\
\hline & $\mathrm{F} 5 \cdots \mathrm{F} 2 \mathrm{a}$ & 2.79 & 140 & 147 \\
\hline & $\mathrm{F} 5 \mathrm{a}^{\cdots} \cdots \mathrm{F} 2$ & 2.68 & 148 & 118 \\
\hline III (MA) & $\mathrm{F} 4 \mathrm{a} \cdots \mathrm{F} 6 \mathrm{a}(\mathrm{F} 3 \mathrm{a})$ & $2.77(2.79)$ & $171(167)$ & $113(110)$ \\
\hline III (MB) & $\mathrm{F} 3 \mathrm{~b} \cdots \mathrm{F} 5 \mathrm{~b}$ & 2.84 & 149 & 109 \\
\hline & $\mathrm{F} 1 \mathrm{~b} \cdots \mathrm{F} 6 \mathrm{~b}$ & 2.88 & 141 & 116 \\
\hline
\end{tabular}
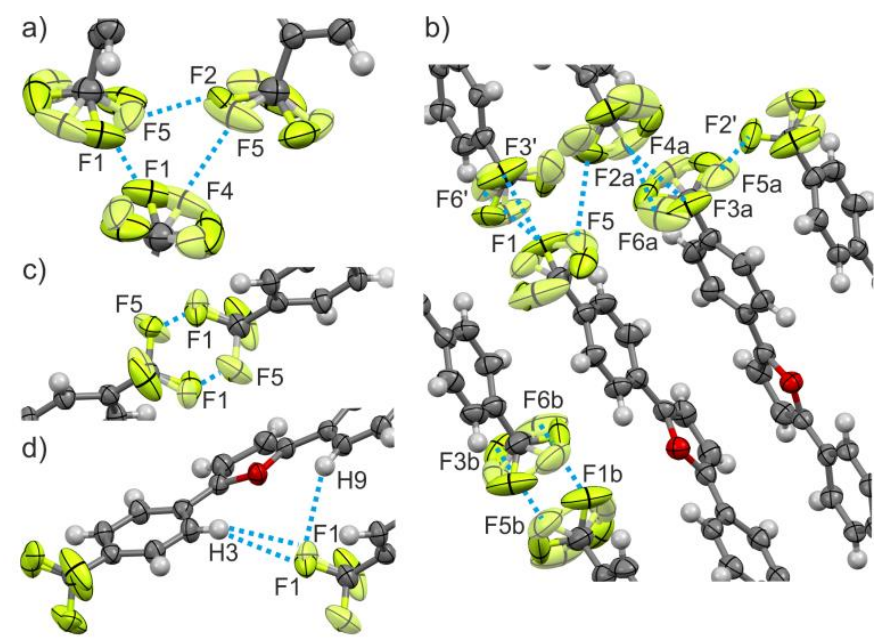

Figure S5 Noncovalent F ...F interactions for disordered $\mathrm{CF}_{3}$ groups of form I (a), form II (c, d) and form III (b). 